\title{
Kartendigitalisierung - buntes Bild oder Mehrwert
}

\section{Digitization of maps - a colourful picture or added value}

\author{
Wolfgang Crom; Berlin
}

Für die Digitalisierung von alten Landesaufnahmen und deren weitere elektronische Aufbereitung arbeitet die Kartenabteilung der Staatsbibliothek mit Kooperationspartnern zusammen, um neuartige Präsentationsformen der einzeln gescannten Kartenblätter zu gewährleisten. Die aufwendige Georeferenzierung erlaubt eine elektronische Montage zum Gesamtkartenwerk und somit ein blattschnittfreies Navigieren. Anhand von drei Beispielen werden Möglichkeiten und Chancen der digitalen Weiterverarbeitung alter Kartenbestände vorgestellt.

Schlüsselwörter: Digitalisierung, alte Landesaufnahmen, Georeferenzierung, Transformation, GIS, WebMapServices

For the digitization of old land survey maps and their digital processing, the map department of the Staatsbibliothek cooperates with partners in order to create a new presentation of the scanned map sheets. The elaborate georeferencing allows an electronic seamless map presentation with an easy navigation. On the basis of three examples possibilities and opportunities of the digital processing will be discussed.

Keywords: digitising, old land surveys, georeferencing, transformation, GIS, WebMapServices

\section{Einführung}

Die zeit- und ortsunabhängige Bereitstellung von wissenschaftlichen Quellen ist mittlerweile eine allgemeine und gut begründete Forderung an Archive, Bibliotheken, Institute oder Museen. Zwar bietet der Bildschirm nur einen begrenzten Ersatz des Originals, der nicht nur das haptische Erfassen vermissen lässt, sondern insbesondere bei großen Objekten wie Karten auch nur eine verkleinerte Ansicht der gesamten Vorlage erlaubt. Insbesondere tritt bei kartographischen wie bei allen bildhaften Materialien jedoch die Besonderheit, nämlich die Gleichzeitigkeit des Überblicks bei der Vertiefung ins Detail, zugunsten eines gesteigerten Vergrößerns von Ausschnitten zurück. Somit ergänzt das elektronische Duplikat die Betrachtung des Originals um diesen sehr nützlichen Aspekt. Im Internet sind inzwischen viele digitale Sammlungen mit kartographischen Materialien eingestellt, die eine bequeme Sicht oder Analyse räumlicher bzw. räumlich-zeitlicher Phänomene erlauben. Auch die hierfür zur Verfügung stehenden Viewer werden immer komfortabler und leistungsfähiger. Doch die Anhäufung von einzelnen Bildern oder Karten in elektronischen Sammlungen oder Datenbanken hat nur einen begrenzten Wert, der die heutigen technischen Möglichkeiten nicht annähernd ausnutzt. Dies gilt insbesondere für Kartenserien, deren Wert und
Zweck aber in der Zusammensetzung zu großflächigen Landschaftsausschnitten oder sogar in der Überlagerung mehrerer Informationsebenen (Inhalt und/oder Zeit) liegt. Unter diesem Aspekt werden drei Projekte unter Beteiligung der Kartenabteilung der Staatsbibliothek zu Berlin vorgestellt, die von externen Kooperationspartnern durchgeführt wurden.

Die digitale Aufbereitung, wie beispielsweise die aufwendige Bearbeitung der Georeferenzierung und Transformierung, kann kaum von Kartensammlungen in der Trägerschaft von Bibliotheken oder Archiven selbst durchgeführt werden. Einerseits fehlt das hierfür fachlich ausgebildete Personal, also Kartographen oder Vermessungsingenieure, andererseits aber auch die Ausstattung mit entsprechender Hard- und Software, um komplexe Vektorberechnungen durchzuführen. So bleibt nur der Weg nach draußen, indem entweder die entsprechende Dienstleistung eingekauft wird oder indem Kooperationen mit anderen Einrichtungen gesucht werden, für die einfache Verortung von Einzelkarten kommt neuerdings auch das Crowdsourcing mit vielversprechenden Ergebnissen in Betracht (Christoph et al 2016). Die Kartenabteilung der Staatsbibliothek zu Berlin hat in den vergangenen Jahren verschiedene Wege beschritten, die an die Projektziele und die Möglichkeiten der Kooperationspartner angepasst waren und die für die
Bibliothek ohne Kosten durchgeführt werden konnten. Die Hauptaufgabe lag für die Kartenabteilung zumeist in der Bereitstellung der digitalen Daten, der Projektberatung und in der Formulierung von Zielvorgaben.

\section{Projekt 1: Der digitale Wenkeraltas}

Georg Wenker (1852-1911) versuchte als Sprachwissenschaftler und Bibliothekar die regionalen Sprachunterschiede im Deutschen Reich zu kartieren. Hierfür entwickelte er umfangreiche Fragebögen, die er mit der Bitte an alle Dorfschullehrer versandte, diese im örtlichen Dialekt zu beantworten. Der Erhebungszeitraum für den beabsichtigten Sprachatlas des Deutschen Reichs lag zwischen den Jahren 1876 und 1887, während dieser Zeit wurden die Fragebögen an über 40.000 Orte gesandt. Die Auswertung der ausgewählten Begriffe und Standardsätze übertrug er in ein einheitliches Kartenmuster. Hierfür wählte er das Indexblatt der Topographischen Karten Deutschlands 1:25.000, das selbst im Maßstab 1:1 Mio. vorlag. So entstanden zwischen 1888 und 19231.668 handgezeichnete Kartenblätter des bis heute weltweit umfangreichsten Sprachatlas, für dessen Ausgestaltung Wenker mit vielen Symbolen und bis zu 22 Farben arbeitete. Aus technischen wie finanziellen Gründen konnte zwischen 1926 und 1956 nur ein kleiner Teil der Daten als Lie- 
ferungswerk und auch nur schwarz-weiß im Druck veröffentlicht werden.'

Im Rahmen eines von der Deutschen Forschungsgemeinschaft finanzierten Projektes konnte 2001 am Institut für Deutsche Sprache an der Universität Marburg an der Lahn mit der digitalen Aufbereitung der in Marburg und Berlin archivierten originalen handgezeichneten Wenker-Karten begonnen werden. Jede Karte besteht aus 3 Teilen, einem Nordwest-, einem Südwest- und einem Nordostblatt, die nach dem Scannen zunächst jeweils digital zu einem Ganzen zusammengefügt wurden. Bestandteil des Projekts Digitaler Wenkeratlas ${ }^{2}$ war auch die Georeferenzierung der Karten, die an den Schnittpunkten der Gitternetzlinien des Indexblattes erfolgte, sowie die Einbindung von Zusatzkarten aus späteren, regionalen Erhebungen. Dadurch sind beispielsweise Hinweise auf Sprachwandel aus den Karten abzulesen. Aber auch andere Karten zur Soziodemographie, Verkehrsinfrastruktur oder Höhenmodelle stehen für die Analyse der Sprachentwicklung zur Verfügung (Herrgen 2007 a; Rabanus 2005; Kehrein et al. 2005). Inzwischen ist die Förderung dieses Projekts abgeschlossen, doch wurde von der Akademie der Wissenschaften und der Literatur Mainz die Finanzierung eines Langzeitprojekts für die intensive Analyse der Daten übernommen. Unter der Bezeichnung $R E D E^{3}$ entsteht ein GIS-basiertes OnlinePortal für die Erforschung regionalsprachiger Phänomene mit einem umfangreichen Aufgabenspektrum, das für Forschung und Öffentlichkeit eine einmalige Plattform bietet und vielfältige Ansätze für neue Fragestellungen bereithält. Nicht nur der Einsatz von Kartenebenen, sondern auch die Verknüpfungen zu den Erhebungsbögen, zu originalen Tonaufnahmen oder bibliographischen Hinweisen bieten ein gänzlich neues, digitales Forschungsumfeld (Abb. 1). Damit werden die Interpretations- und Aussagemöglichkeiten des Originals durch den Einsatz moderner Techniken erheblich vergrößert (Herrgen 2007 b; Lameli 2013).

\section{Alte Landesaufnahmen}

Topographische Kartenwerke können als die hohe Schule der Kartographie angesprochen werden. Die enge Verzahnung der präzisen Vermessung und das Können der graphischen Wiedergabe lassen in ihnen ein generalisiertes, aber exaktes Bild der Welt entstehen, das mit einem besonderen Symbolschlüssel der Legende übersichtlich gestaltet und somit gut und schnell lesbar ist. Nicht

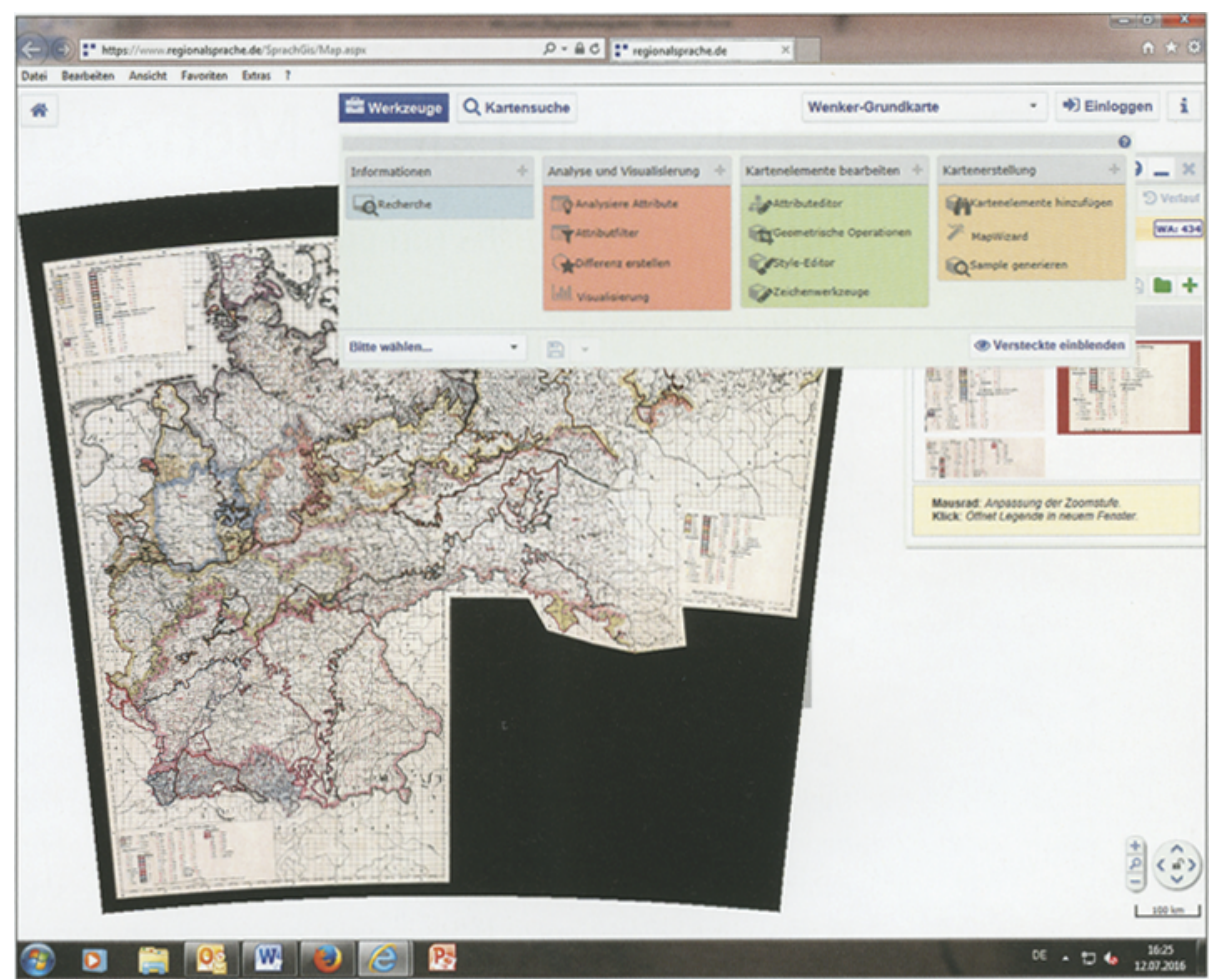

Abb. 1: Der Wenker-Atlas im GIS-Portal Regionalsprache.de (Beispiel Karte 434 bisschen)

ohne Grund wird die Herstellung dieses Kartentypus als hoheitliche Aufgabe betrachtet und so sind amtliche oder sogar militärische Stellen damit beauftragt, ein getreues Abbild der Erde zu schaffen und dabei das Problem der Wiedergabe einer Dreidimensionalität auf einem Blatt Papier oder einem Bildschirm zu minimieren. Definitorisch werden sie als ortsbeschreibende Karte, die eine Landschaft dem jeweiligen Maßstab entsprechend vollständig und geometrisch korrekt wiedergibt, beschrieben. Zum Inhalt gehört eine Geländedarstellung, ferner die lagerichtige Eintragung von Gewässern, Vegetation, Siedlungen, Verkehrswegen und sonstigen Geländemerkmalen, die als Situationsdarstellung bezeichnet wird. Stete Veränderungen in dicht besiedelten Räumen machen eine dauerhafte Fortführung der vermessungstechnischen und kartographischen Arbeiten notwendig. Größere zusammenhängende Gebiete müssen dabei in einer Vielzahl von einheitlichen Kartenblättern im Regelblattschnitt erstellt werden.

Die Anfänge dieses Kartentyps liegen im 18. Jahrhundert, als die Trigonometrie allmählich zur Grundlage der exakten Landesvermessung wurde. Viele Staaten experimentierten auf diesem Feld, aus denen im Verlauf des 19. Jahrhunderts feste Regeln und schließlich Normungen abgeleitet wurden, auf denen die hohe Qualität der heutigen Kartenwerke fußt. Doch bis dieser Standard erreicht werden konnte, waren viele Schritte notwendig, die sich in den alten Landesaufnahmen widerspiegeln. Diese Kartenwerke sind heute eine unverzichtbare Quelle für landesgeschichtliche Forschungen, wie ihre tägliche Nutzung beweist, auch wenn sie noch viele Fehler enthalten.

Die Digitalisierung und Onlinepräsentation der vielen einzelnen Kartenblätter eines Kartenwerks ist außer der zeit- und ortsunabhängigen Verbreitung allerdings nicht sehr befriedigend, denn durch den vorgegebenen Blattschnitt wird immer nur ein Ausschnitt wiedergegeben. Zumeist erstreckt sich aber das zu betrachtende Gebiet über mehrere Kartenblätter. In einer Kartensammlung mit großem Tisch können die analogen Blätter zu einer großen Einheit zusammengelegt werden, die Betrachtung einzelner Bilder auf dem Bildschirm bietet diesen Vorteil nicht. Die blattschnittfreie Präsentation der gesamten Fläche einer alten Landesaufnahme mit einer frei beweglichen Navigation ist daher ein Desiderat,

\footnotetext{
Die Teilpublikation von 128 Karten erschien als Deutscher Sprachatlas (DSA) zwischen 1927 und 1956 unter der Bearbeitung von Ferdinand Wrede, Walther Mitzka und Bernhard Martin.

2 DiWA: http://www.diwa.info. Weiterführende Informationen auch unter: http://de.wikipedia. org/wiki/Deutscher_Sprachatlas

3 I. e.: Regionalsprache.de http://www.regional sprache.de
} 
dessen Machbarkeit die Kartenabteilung der Staatsbibliothek zu Berlin in zwei weiteren Kooperationsprojekten testen konnte.

Doch wie sieht es bei alten topographischen Landesaufnahmen aus? Können alte Kartenwerke in dieser Form aufgearbeitet werden und welcher Aufwand muss betrieben werden? Und könnten diese vektorisierten $\mathrm{Da}$ ten mit dem heutigen geodätischen Muster überhaupt in Übereinstimmung gebracht werden, damit eine diachronische Betrachtung der Landschaftsentwicklung präsentiert werden kann? Diesen Fragen ist die Kartenabteilung nachgegangen und konnte für die Beantwortung verschiedene Projektpartner finden.

\subsection{Projekt 2: Sächsische Meilenblätter}

In den Jahren 1780 bis 1806 fand die erste flächendeckende Kartierung des Kurfürstentums Sachsens statt. Mit der Ausführung wurde das Ingenieurkorps unter der Leitung von Major Friedrich Ludwig Aster (17321804) beauftragt. Ausgehend von der Basislinie bei Pirna wurde mit der trigonometrischen Vermessung begonnen, die Kartierung erfolgte mit der Messtischmethode. Die Geländeaufnahmen wurden per Einschneideverfahren durchgeführt, für die Darstellung in den Karten kam später die Schraffenmanier nach Johann Georg Lehmann (1765-1811) zur Anwendung. Da die Basislinie gleichzeitig als Blattgrenze diente, ist das Kartenwerk nicht genordet, sondern um $42^{\circ}$ nach Westen gedreht. Die Blattgröße bemisst eine Quadratelle und zeigt das Gebiet einer Quadratmeile, wovon sich der Name Meilenblätter ableitet. Das Verhältnis von Dresdner Elle zur kleinen sächsischen Meile ergibt den Maßstab 1:12.000 (Brunner 2002; Brunner 2005; Moser 2013). Von den Originalblättern, die heute im Sächsischen Hauptstaatsarchiv liegen, wurden jeweils unmittelbar nach ihrer Fertigstellung Kopien für das Herrscherhaus angefertigt, die nach der Völkerschlacht bei Leipzig 1813 an Preußen ausgeliefert wurden und sich seit 1919 im Bestand der Kartenabteilung der Staatsbibliothek zu Berlin befinden. Diese Berliner Ausgabe genannte Kopie hat einen Umfang von 371 Kartenblättern und ist die am besten erhaltene Version, die 2006 als Grundlage für die Digitalisierung in Kooperation mit Sächsischen Landesbibliothek Staats- und Universitätsbibliothek Dresden diente. Sie werden seitdem im Kartenforum Sachsen der Deutschen Fotothek als Einzelblätter präsentiert. ${ }^{4}$

Gleichzeitig wurde mit der Hochschule für Technik und Wirtschaft, Fakultät Geoinformation in Dresden, ein weiterer Kooperationspartner gewonnen. Unter der Leitung von Frau Prof. Müller wurde mit Studierenden die Georeferenzierung der Meilenblätter durchgeführt. Zunächst musste hierfür das historische System rekonstruiert werden, um es mit dem heutigen Referenzsystem in Übereinstimmung bringen zu können. Als Grundlage wurden die Schnittpunkte des auf den Kartenblättern eingezeichneten Gitternetzes herangezogen. Bereits 2007 lag eine

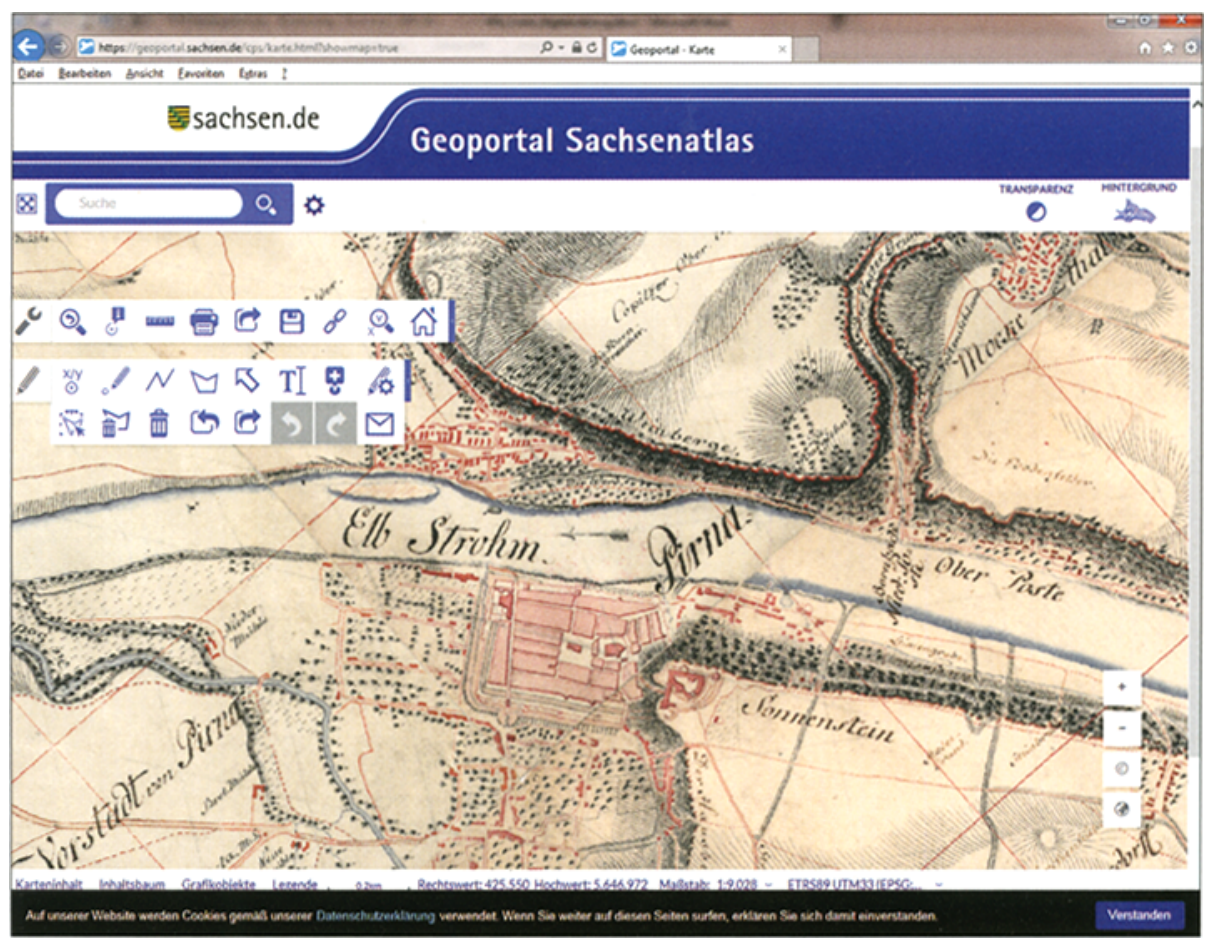

erste Diplomarbeit über den Workflow für die Einbindung in einen Web Map Service vor (Muschter 2007). Auf dem Server der Hochschule entstand erstmalig eine Webbrowser-versierte, für die Öffentlichkeit frei zugängliche Version der blattschnittfreien Darstellung des gesamten Kartenwerkes mit Zoomfunktion für die Betrachtung einzelner Details. Inzwischen ist das Kartenwerk in den Datenpool des Geoportals Sachsenatlas ${ }^{5}$ integriert und mit vielfältigen Anwendungsmodulen (Abb. 2) zu einer wichtigen Arbeitsgrundlage für die Landesplanung geworden (Müller 2009). Auch der Themenstadtplan Dresden ${ }^{6}$ hat die Daten in seine Anwendung als Hintergrundkarte übernommen.

Die Verbindung des alten Referenzsystems mit dem heutigen impliziert eine Drehung der Meilenblätter auf die Nordrichtung. In der Darstellung ist dadurch die ursprünglich waagerecht verlaufende Schrift aufwärtsgerichtet. Die Einbindung von freien Daten, eine stufenlos regelbare Transparenz von Karten sowie die Verfügbarkeit von Zeichenund Standardwerkzeugen machen das Geoportal mit den historischen Karten zu einem vielseitigen Instrumentarium für angewandte Landesgeschichtsforschung. Die Bedeutung alter Landesaufnahmen für eine kartenbasierte Landschaftsentwicklung wurde einige Jahre zuvor bereits am Leibniz-Institut für Ökologische Raumentwicklung in Dresden hervorgehoben, indem für die interne Datengrundlage verschiedene Landeskartenwerke in einem System zu einer diachronischen Betrachtung für den sächsischen Raum eingearbeitet wurden und seitdem die wesentliche Grundlage für vielfältige Fragen zur Kulturlandschaftsgenese bilden. Hierzu wurden auch die sächsischen Meilenblätter hinzugezogen, georeferenziert und transformiert (Witschas 2002; Walz et al. 2011).

\subsection{Projekt 3: Schmettausches Kartenwerk}

Das dritte und jüngste Projekt ist im Ergebnis vergleichbar mit dem zweiten hier vorgestellten Vorhaben, es entstand jedoch auf Initiative des Landesbetriebs Forst Branden-

\footnotetext{
http://www.deutschefotothek.de/cms/karten forum-sachsen-meilenblaetter-berlin.xml

5 http://geoportal.sachsen.de s. Karteninhalt: Auswahl 04/13 Historisches Sachsen und Checkbox Meilenblätter Sachsen.

6 http://stadtplan.dresden.de s. Ordner Hintergrundkarte und Checkbox Berliner Meilenblätter.
} 

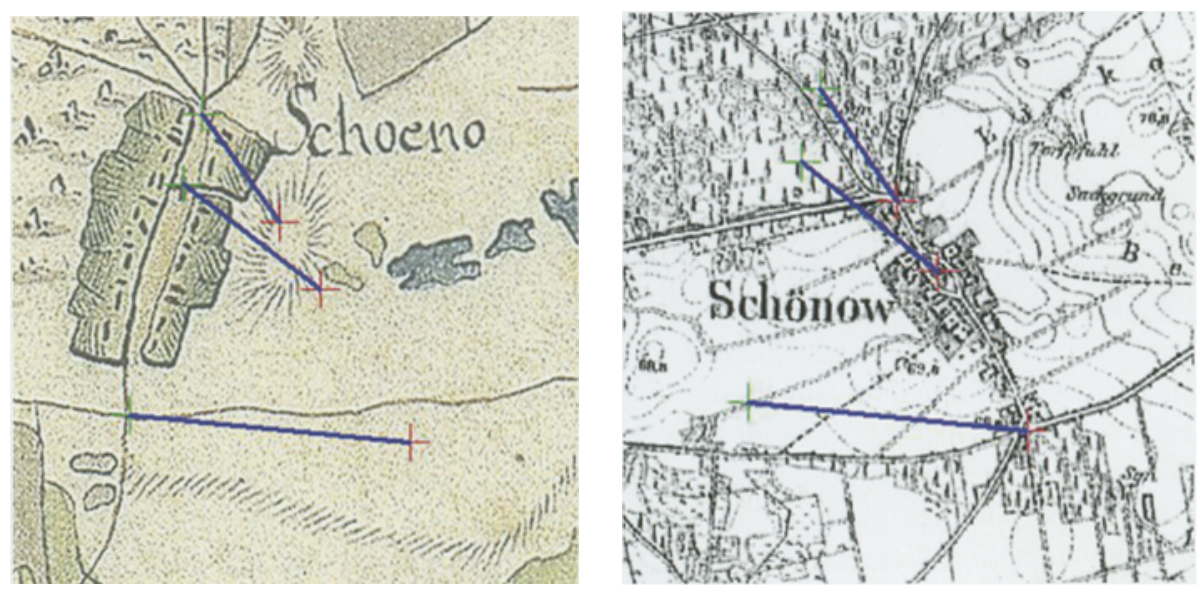

Abb. 3: Schmettausches Kartenwerk: Bestimmung der Lagegenauigkeit des Ortes Schönow: grünes Kreuz = Passpunkt Schmettau, rotes Kreuz = Passpunkt Messtischblatt, blau = Vektor

burg mit einer konkreten Nutzanwendung. Für die Ermittlung der Waldstandorte und Waldflächen des Landes Brandenburg im ausgehenden 18. Jahrhundert sollten Karten der ersten flächendeckenden Kartierung Preußens im Maßstab 1:50.000 ausgewertet werden, die unter der Leitung des Grafen Friedrich Wilhelm Carl von Schmettau (1743-1806) zwischen 1767 und 1787 entstanden waren. Die Entstehung der insgesamt 270 Sektionen erfolgte dabei ohne die Grundlage eines trigonometrischen Netzes (Flint, Jordan 2008). Neben einer Kartierung im Gelände sind wohl auch vorhandene Vorlagen verwendet worden, die nach Vorgabe für das Kartenwerk umgezeichnet wurden. Dennoch bezeichnete es Scharfe als „... in Inhalt wie in Umfang das Beste (...), was für Brandenburg-Preußen bis zu dieser Zeit an topographischen Karten vorgelegt werden konnte" (Scharfe 1982, S. 1). Die Fläche des heutigen Bundeslandes Brandenburg wird von 32 Blättern abgedeckt, die für

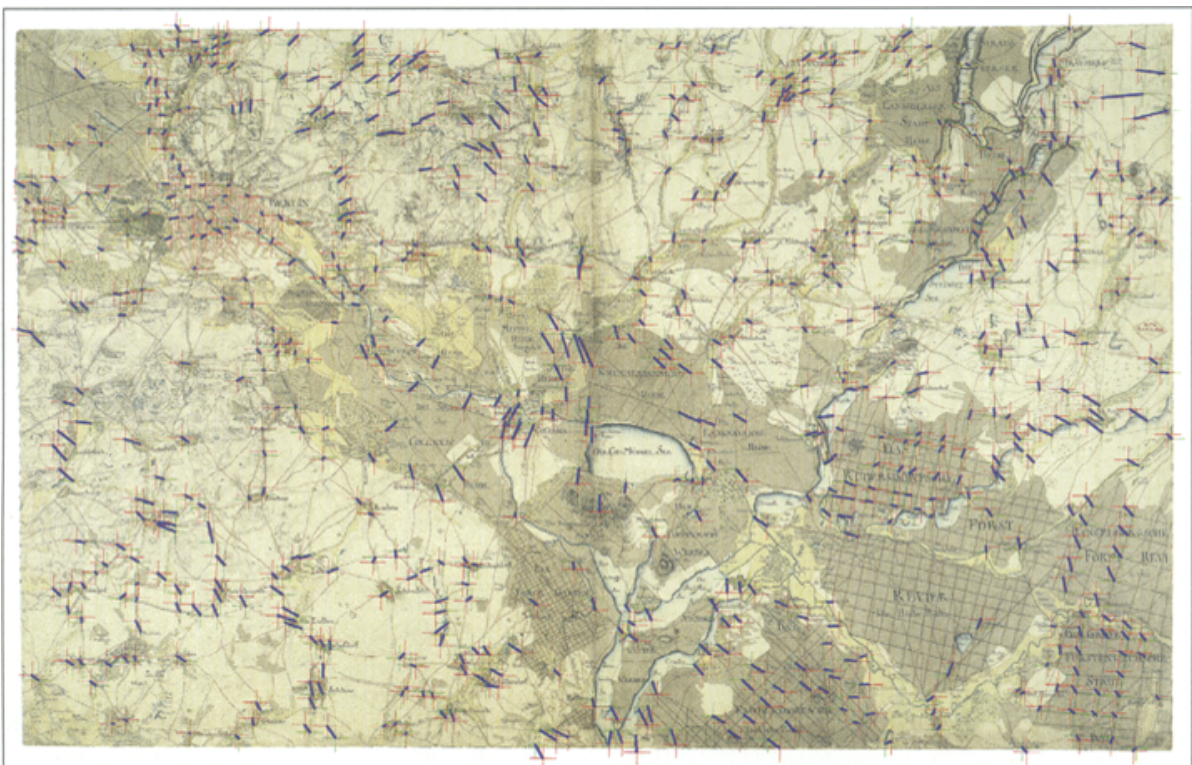

Abb. 4: Schmettausches Kartenwerk, Passpunkte und Vektoren für das Blatt Berlin wurde dagegen der Landesvermessung Geobasisinformation Brandenburg übergeben, um sie für die Einspielung in den Brandenburgviewer aufzubereiten. Damit ist auch dieses Kartenwerk für die Öffentlichkeit frei zugänglich, allerdings noch nicht mit einer optimalen Auflösung. Doch anhand dieses Beispiels soll der Aufwand für die Georeferenzierung und Transformation einer Landesaufnahme ohne Trigonometrie dargestellt werden.

Hierzu waren verschiedene Arbeitsschritte notwendig, womit das Landesamt Forst Brandenburg als Projektträger das Vermessungsbüro Jörg Schröder in Guben beauftragte, sodass die folgenden Ausführungen auf diesen Arbeiten beruhen (Schröder 2012 und 2013). Da viele Kartenblätter zerschnitten und auf Leinen geklebt sind, mussten zunächst die Beschneidungskanten wieder zusammengefügt und anhand von Verknüpfungslinien ausgerichtet und angepasst werden. Im zweiten Arbeitsschritt wurden Passpunkte in Ortslagen gesucht, die damals wie heute in den Karten zu finden sind. In erster Linie eigneten sich hierzu Kirchen, Stadttore oder Schlossanlagen. Zur Überraschung musste festgestellt werden, dass es große Abweichungen in der Lagegenauigkeit gab, in einzelnen Fällen war sogar die gesamte Ortslage verdreht: Statt seiner tatsächlichen Ausrichtung Nordwest-Südost ist beispielsweise der Ort Schönow im Schmettauschen Kartenwerk in einer Nordnordost-Südsüdwest-Lage dargestellt (Abb. 3). Weitere Passpunkte wurden im freien Gelände gesucht, die ebenfalls in heutigen Karten noch nachgewiesen sein mussten, wie Teeröfen, Mühlen, Brücken oder Wegekreuzungen, sodass pro Kartenblatt möglichst viele Vektoren für eine Umrechnung zur Verfügung standen (Abb. 4). Um die Übergänge zu den Nachbarblättern möglichst exakt zu gewährleisten, wurden alle Randpunkte erfasst. Durch die Transformation der Vektoren wurden die Kartenblätter verzerrt (Abb. 5) und konnten anschließend elektronisch zusammengefügt werden. Das nun im Brandenburgviewer der Landesvermessung und Geobasisinformation Brandenburg ${ }^{8} \mathrm{zu}$ betrachtende Ergebnis wird mit einer noch geringen Auflösung in der Darstellung der historischen Landesauf-

\footnotetext{
7 http://www.brandenburg-forst.de/webgis s. Ordner: Historische Waldkartierungen und Checkbox Wald nach Schmettau.

8 http://bb-viewer.geobasis-bb.de/ s. Kartenebenen/Geofachdaten: Historische Daten Checkbox Schmettaukarten.
} 


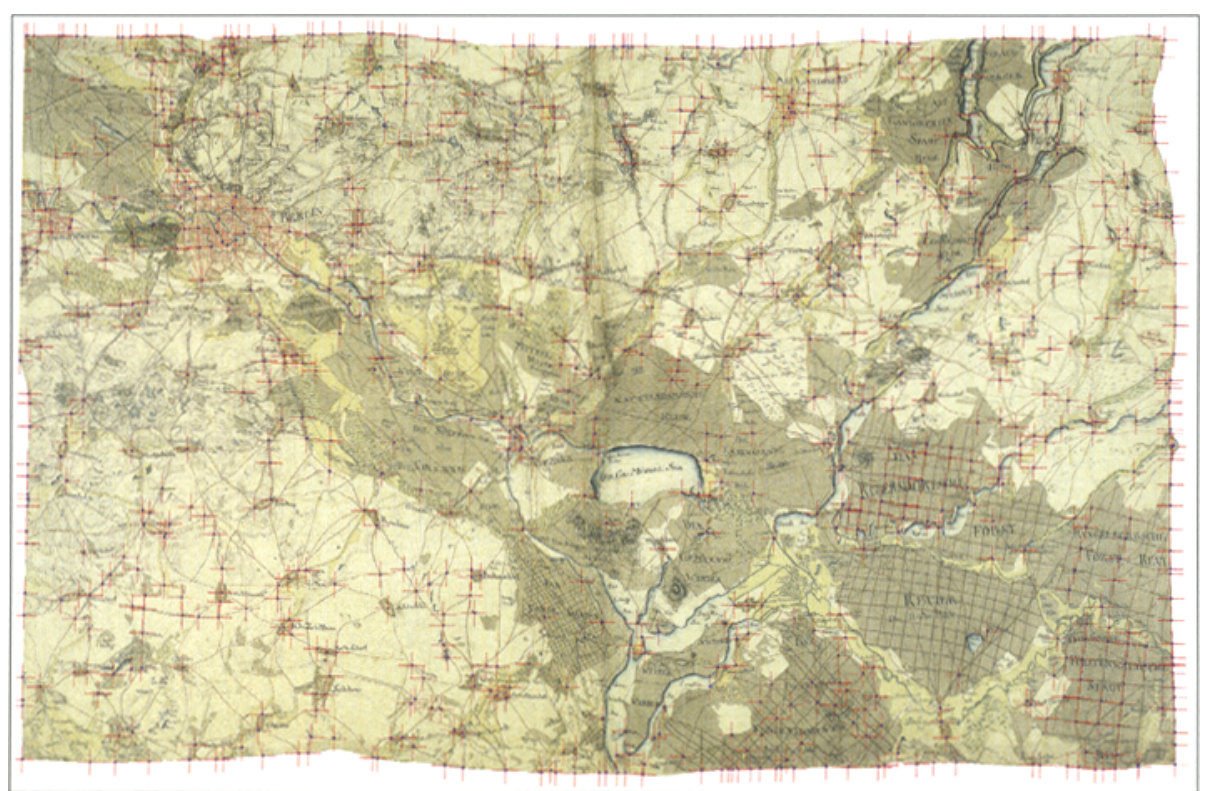

Abb. 5: Schmettausches Kartenwerk, Ergebnis nach der Vektortransformation für das Blatt Berlin

nahme von Schmettau angeboten. Die für Geoportale inzwischen üblichen Werkzeuge für Strecken- und Flächenmessung oder Ortssuche sowie zuschaltbare Kartenebenen sind ähnlich wie im Geoportal des Sachsenatlas verfügbar.

\section{Zusammenfassung}

Die digitale Aufbereitung gescannter Kartenblätter und die vektorielle Umrechnung auf heutige geodätische Systeme für eine Einbindung in GIS-gesteuerte Programme ist nur mit einem erheblichen Zeitaufwand, historisch-geodätischem Wissen, fachlicher Qualifikation und entsprechenden finanziellen Mitteln zu leisten. Allein das Projekt Sächsische Meilenblätter mit einer Hochschule als Kooperationspartner konnte ohne externe Finanzierung durchgeführt werden. Als Versuchsballon im Einsatz der studentischen Ausbildung waren keine Personalkosten entstanden, das erreichte Ziel hat jedoch alle Erwartungen erfüllt und damit die Mühen gelohnt. Die Anfertigung von Examensarbeiten ist dabei nicht nur eine Begleiterscheinung, sondern kann als Motor für den Einsatz innovativer Methoden und einer stetigen Anpassung gesehen werden. Für Öffentlichkeit und Wissenschaft öffnen sich mit Web Map Services neue Perspektiven. Das erste Projekt, der Digitale WenkerAtlas, ist inzwischen die Basis in einem großen Folgeprojekt; die Sprachwissenschaftler haben damit ein komplett neues Arbeitsinstrument mit ungeahnten Forschungsfeldern erhalten und nutzen es sehr intensiv weit über die Grenzen des Instituts für Deutsche Sprache hinaus. Alte Landes- aufnahmen werden dagegen nicht nur von Wissenschaftlern genutzt, sondern insbesondere von angewandten Behörden und von einer breiten Gruppe an Heimatforschern, die an früheren Landschaftszuständen interessiert sind. Für angewandte Spezialanwendungen beispielsweise im Forstwesen, in der Archäologie, im Naturschutz oder in der Regionalplanung bringen die neuen technischen Möglichkeiten unmittelbaren Nutzen. Nicht die quantitative Menge des inzwischen digitalisierten Kartenbestands von ca. 30.000 Blättern aus der Kartenabteilung der Staatsbibliothek zu Berlin, die ebenfalls beeindruckend ist, sondern die qualitative und anwendungsorientierte Aufarbeitung stehen im besonderen Fokus unserer Aktivitäten, die wir jedoch nur mit verlässlichen Partnern zu leisten in der Lage sind.
Anzeige
Literatur

Brunner, H. (2002): Wie Sachsen vermessen wurde: die Meilenblätter und die kursächsische Landesvermssung von 1780 bis 1825 ; eine Geschichte der topographischen Kartographie in Sachsen. Dresden: Hochschule für Technik und Wirtschaft (= Dresdner Kartographische Schriften ; 5)

Brunner, H. (2005): Die sächsische Landesaufnahme von 1780 bis 1825 . Leipzig : Sächsische Akademie der Wissenschaften zu Leipzig. (= Atlas zur Geschichte und Landeskunde von Sachsen; Reihe H: Historische Karten ; 12.1/12.2, Beih.)

Christoph, A.; Ammon, M.; Crom, W.; Przibytzin, H. (2016): Digitalisierung, Verortung, Recherche - Neue Perspektiven für historische Karten. In: Kartographische Nachrichten 66, S. 115-122

Flint, O. und Jordan L. (2008): Friedrich Wilhelm Carl von Schmettau - Pionier der modernen Kartographie, Militärschriftsteller, Gestalter von Parks und Gärten. Frankfurt (Oder)

Herrgen, J. (2007a): Dialectology - Digital and Interactive: The Digital Wenker Atlas - DiWA. In: The National Institute for Japanese Language: Geolinguistics around the World. Proceedings of the $14^{\text {th }}$ NIJL International Symposium. Tokyo, August $22-23,2007$, S. $29-34$

Herrgen, J. (2007b): From dialect to variation space: The "Regionalsprache.de" (REDE) Project. In: The National Institute for Japanese Language: Geolinguistics around the world. Proceedings of the $14^{\text {th }}$ NIJL International Symposium. Tokyo, August 2223, 2007, S. 75-80

Kehrein, R.; Lameli, A.; Nickel, J. (2005): Möglichkeiten der computergestützten Regionalsprachenforschung am Beispiel des Digitalen Wenker-Atlas (DiWA). In: Jahrbuch für Computerphilologie 7 , S. 149-170 (http://www.computerphilologie.unimuenchen.de/jg05/kehrein/kehrein.html)

Lameli, A. (2013): Strukturen im Sprachraum. Analysen zur arealtypologischen Komplexität der Dialekte in Deutschland. Berlin (= Linguistik - Impulse \&t Tendenzen ; 54)

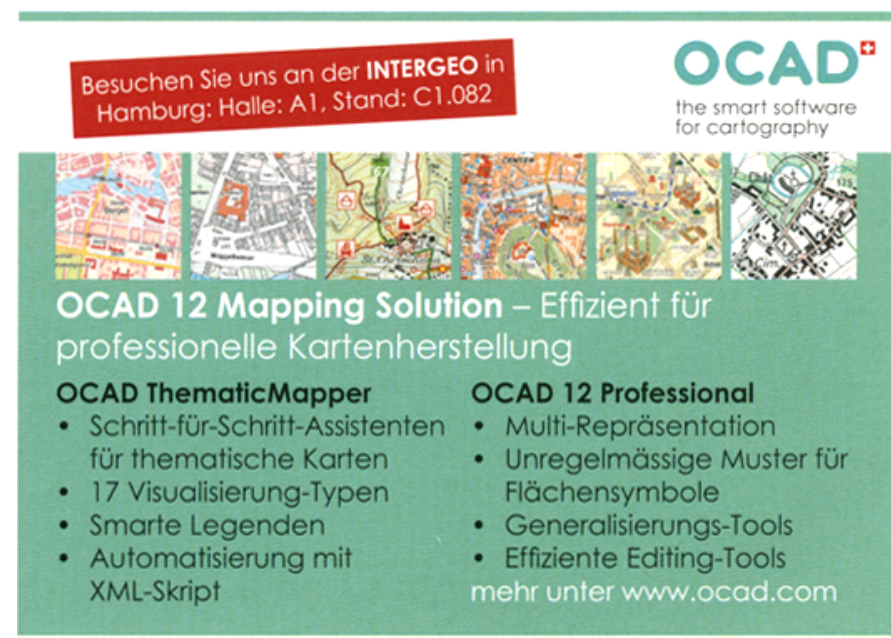


Moser, J. (2013): Die Besonderheiten der Kartenproduktion in Sachsen im Rahmen der Mitteleuropäischen Gradmesseung (seit 1862). In: Vermessung Brandenburg [18], S. 31-35 (http://www.geobasisbb.de/GeoPortal1/produkte/verm_bb/pdf/1_13_ Moser_31-35.pdf)

Müller, M. (2009): Alte Landkarten in neuem Gewand. Der Web Map Service - Berlin Meilenblätter Sachsens. In: Sächsische Heimatblätter 55, S. 114121

Muschter, T. (2007): Workflow für einen UMN-WMS für flächendeckende historische Kartenwerke. Hochschule für Technik und Wirtschaft Dresden, unveröffentlichte Diplom-Arbeit

Rabanus, S. (2005): Sprachkartographie des Deutschen: von Schmeller bis zum Digitalen Wenker-Atlas. In: Perspektiven Eins : Akten der 1. Tagung Deutsche Sprachwissenschaft in Italien. S. 345-362 (http://www.dfll.univr.it/documenti/ OccorrenzaIns/matdid/matdid213717.pdf)

Scharfe, W. (1982): Berlin und Umgebung 1774/1775. Vier Blätter des Schulenburg-Schmettauschen Kartenwerks. Lüneburg ( $=$ Quellen zur Geschichte der deutschen Kartographie ; 3)

Schröder, J. (2012): Projektbericht: Digitalisierung historischer Waldgebiete, Preußische Landesaufnahme 1877-1915, Schmettau'sches Kartenwerk 17671787. Bearb. im Auftrag des Landesbetriebs Forst Brandenburg [unveröffentlicht]

Schröder, J. (2013): Georeferenzierung und Entzerrung der brandenburgischen Kartenblätter des Schmettauschen Kartenwerks. In: Vermessung Brandenburg [18], S. 14-23 (https://www.geobasis-bb.de/ verm_bb/pdf/2_13_Schroeder_14-23.pdf)

Walz, U.; Schumacher, U. (2011): Sächsische Meilenblätter als Quelle der Kulturlandschaftsforschung am Beispiel der Sächsischen Schweiz. In: Cartographica Helvetica 44, S. 3-15 (http://www.e-periodica.ch/ digbib/view?pid=chl-001:2011:43-44::114\# 114)

Wenker, G. (2013): Schriften zum „Sprachatlas des Deutschen Reichs“. Gesamtausgabe. Herausgegeben und bearbeitet von Alfred Lameli. Unter Mitarbeit von Johanna Heil und Constanze Wellendorf. 3 Bände. Hildesheim (= Deutsche Dialektgeographie; 111)

Witschas, S. (2002): Erinnerung an die Zukunft: sächsische historische Kartenwerke zeigen den Landschaftswandel. - In: Kartographische Nachrichten 52, S. 111-117

Über den Autor

Wolfgang Crom leitet die Kartenabteilung der Staatsbibliothek zu Berlin - Preußischer Kulturbesitz. Kontakt: wolfgang.crom@sbb.spk-berlin.de

Manuskriptveröffentlichung erfolgt auf Einladung der Redaktion.

In dieser Ausgabe finden Sie Beilagen von

CPA Software GmbH 53721 Siegburg

Wir bitten unsere Leser um freundliche Beachtung.

\title{
Geovisualisierung und Text Mining mit Microblogging Content
}

\author{
Geovisualization and Text Mining with \\ Microblogging Content
}

\author{
Mathias Gröbe, Dirk Burghardt; Dresden
}

Soziale Medien wie Twitter und Flickr sind zurzeit beliebte Kommunikationsplattformen, die es Nutzern ermöglichen, Kurznachrichten, Fotos oder Links auszutauschen. Inhalte, die für jedermann sichtbar sind, können über Programmierschnittstellen abgerufen, analysiert und weiterverarbeitet werden. Von besonderem Interesse für die Geowissenschaftler sind Beiträge, deren Metadaten einen Ortsbezug aufweisen. In diesem Artikel wird ein Verfahrensablauf vorgestellt, wie sich aus georeferenziertem Text raumbezogene thematische Informationen unter anderem mittels Text Mining gewinnen und kartographisch darstellen lassen. Visualisierungswerkzeuge können dabei sowohl für semiautomatische Datenaufbereitung als auch für Analyse und Präsentation der extrahierten Informationen verwendet werden. Anhand verschiedener Beispiele wird im Beitrag illustriert, welche Art von Information sich aus den raumbezogenen Daten extrahieren lässt und welche spezifischen Eigenschaften die durch das Kurznachrichtenportal Twitter und die Fotodatenbank Flickr generierten Daten besitzen.

- Schlüsselwörter: Twitter, Flickr, Geovisualisierung, Text Mining

Nowadays, social media platforms such as Twitter or Flickr are increasingly popular. They enable users to share short messages, pictures or web links with the public or friends. Publicly visible content can be accessed using APIs and frameworks, and - after some pre-processing steps - can be used for a variety of different analyses. Postings with geographic meta-data might be of high interest for geo-scientists. This paper deals with a workflow of how to extract and map thematic information associated with a location from geo-referenced text data. The workflow shows that is possible to use tools for Geovisualization for semi-automated information retrieval, information extraction and information presentation. A case study with data from the short messages network Twitter and the photo database Flickr is used to demonstrate how to extract spatial information and illustrate typical characteristics of microblogging content.

- Keywords: Twitter, Flickr, Geovisualization, Text Mining

\section{Einleitung}

Mit der gewachsenen Popularität sozialer Netzwerke und Web-2.0-Plattformen zeigt sich das große Interesse verschiedener Nutzergruppen, aktuelle Informationen zu verschiedensten Themen aus dem persönlichen Erleben und der lokalen Umgebung digital zu erfassen und online zu teilen. Beispiele reichen von OpenStreetMap, über Social-Media-Plattformen bis hin zu digitalen Enzyklopädien. Die dabei generierten Geodaten unterscheiden sich zum Teil stark von amtlichen oder kommerziellen Geodaten insbesondere hinsichtlich Strukturie- rung, Aktualität, Abdeckung, Detaillierungsgrad, Subjektivität etc. und bieten insofern großes Forschungs- und Anwendungspotenzial.

\subsection{Microblogging}

Unter Microblogging versteht man den Austausch von kurzen Informationen, vor allem Texte, aber auch Links, Fotos oder Videos. Als bekanntestes Beispiel für Microblogging Content wird oft Twitter ${ }^{1}$ mit seinem Fokus auf kurze Mitteilungen, den sogenannten „Tweets“, angeführt. Aber

\footnotetext{
1 https://www.twitter.com/
} 